\title{
Superhydrophobic Breakdown of Nanostructured Surfaces Characterized in Situ Using ATR-FTIR
}

\author{
Nandi Vrancken, ${ }^{*}, \dagger$, Stefanie Sergeant, ${ }^{\S, \|}$ Guy Vereecke, ${ }^{\ddagger}$ Geert Doumen, ${ }^{\ddagger}$ Frank Holsteyns, ${ }^{\ddagger}$
}

Herman Terryn, ${ }^{\dagger}$ Stefan De Gendt, ${ }^{+}$and XiuMei $\mathrm{Xu}^{*}, \stackrel{+}{+}$

${ }^{\dagger}$ Vrije Universiteit Brussel, Pleinlaan 2, 1050 Elsene, Belgium

${ }^{\ddagger}$ Imec, Kapeldreef 75, 3001 Leuven, Belgium

${ }^{\S}$ UC Leuven-Limburg, Herestraat 49, 3000 Leuven, Belgium

\section{Supporting Information}

ABSTRACT: In situ characterization of the underwater stability of superhydrophobic micro- and nanostructured surfaces is important for the development of self-cleaning and antifouling materials. In this work, we demonstrate a novel attenuated total reflectance-Fourier transform infrared (ATR-FTIR) spectroscopy-based method for large-area wetting characterization of silicon nanopillars. When air is present in between the structures, as is characteristic of the Cassie-Baxter state, the relative intensities of the water bands in the absorption spectrum change because of the wavelengthdependent attenuation of the evanescent wave. This phenomenon enables unambiguous identification of the wetting state and assessment of liquid impalement. Using

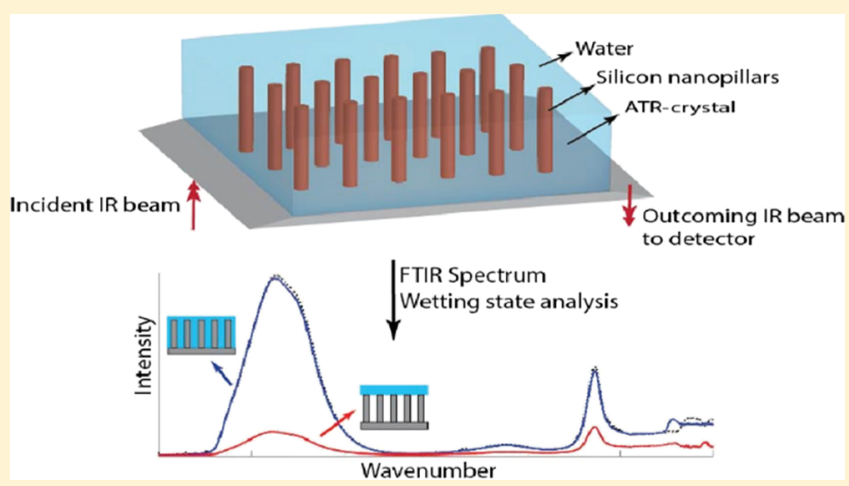
mixtures of isopropanol and water with different concentrations, the breakdown of superhydrophobic states and the wetting hysteresis effects are systematically studied on uniform arrays of silicon nanopillars. A transition from the Cassie-Baxter to Wenzel state is observed when the isopropanol concentration exceeds $2.8 \mathrm{~mol} \%$, corresponding to a critical surface tension of 39 $\mathrm{mN} / \mathrm{m}$. Spontaneous dewetting does not occur upon decreasing the isopropanol concentration, and pure water can be obtained in a stable Wenzel state on the originally superhydrophobic substrates. The developed ATR-FTIR method can be promising for real-time monitoring of the wetting kinetics on nanostructured surfaces.

\section{INTRODUCTION}

When a liquid is brought into contact with a structured surface, the liquid may either fully or partially wet the surface depending on the interfacial energies. Two models are generally referred to when describing the wetting state, as was proposed almost a century ago by Wenzel ${ }^{1}$ and Cassie and Baxter ${ }^{2}$ and have been elaborately described in recent reviews. ${ }^{3-5}$ In the Wenzel model, the liquid fully penetrates the surface structures, and the apparent contact angle measured on the rough surface $\left(\theta_{\mathrm{W}}^{*}\right)$ is given by

$$
\cos \left(\theta_{\mathrm{W}}^{*}\right)=r \cos (\theta)
$$

where $\theta$ is the contact angle on a flat surface of the same material and $r$ is the roughness factor defined as the ratio of the true and projected surface areas, that is, $r=A_{\text {real }} / A_{\text {projected. }}$ The Cassie-Baxter model describes a superhydrophobic state with the liquid residing on top of the structures and an air layer trapped in the surface cavities. The apparent contact angle of a droplet in the Cassie-Baxter state $\left(\theta_{\mathrm{CB}}^{*}\right)$ is given by

$$
\cos \left(\theta_{\mathrm{CB}}^{*}\right)=f_{\mathrm{s}} \cos (\theta)-1+f_{\mathrm{s}}
$$

in which $f_{\mathrm{s}}$ represents the solid surface fraction. Cassie-Baxter states are generally characterized by very high contact angles and a low contact angle hysteresis. Various plant species exploit this property to remove particles and dirt from their leaves, as shown by Barthlott. ${ }^{6}$ Inspired by this observation in nature, applications of superhydrophobic surfaces for self-cleaning and antifouling materials have already been demonstrated. ${ }^{7-9}$ However, breakdown of the superhydrophobicity is generally irreversible $\mathrm{e}^{10,11}$ and limits the lifetime of these surfaces. On the other hand, complete wetting of nanostructured surfaces is desired for applications from wet processing in integrated circuits (IC) fabrication ${ }^{12-15}$ to single molecule detection in nanofluidics. ${ }^{16,17}$ Consequently, establishing an in-depth understanding of the wetting behavior on nanostructured surfaces is critical. The most commonly used method to study solidliquid interactions and superhydrophobic breakdown is based on goniometric techniques, that is, macroscopic contact angle measurements. ${ }^{18-24}$ This technique yields a good estimation of

Received: December 13, 2016

Revised: February 15, 2017

Published: March 24, 2017 
the wetting state in most cases. However, contact angles are dictated by the area in the vicinity of the triple-phase line and do not provide information on the liquid behavior away from the drop edge. ${ }^{25}$ Therefore, close attention should be paid when interpreting data from heterogeneous surfaces, mixed wetting states, or wetting transitions. Moreover, contact angle measurements can be affected by experimental parameters, for example, drop size, dispensing rate, applied pressure, and so forth.

Various other techniques have been developed to obtain more detailed information on the liquid-solid interactions on the micro- or nanoscale. The high number of very recent publications ${ }^{13,26-31}$ illustrates the relevance and interest in this field. Techniques for wetting state characterization can be classified according to the length scale of the surface roughness, that is, microstructured or nanostructured surfaces (characteristic dimension below the optical diffraction limit). Analysis of wetting states on microstructures is commonly based on optical methods. Conventional microscopy allows for differentiation between the Wenzel and Cassie-Baxter states by evaluation of the droplet shape and image focus. ${ }^{32,33}$ Recent works ${ }^{28,31,34-36}$ have reported application of in situ confocal microscopy to visualize the liquid interface on micropillars and inside of micropores. Additionally, accurate 3D-imaging of the liquid interface and assessment of local liquid impalement are feasible with interference microscopy. ${ }^{37}$ Lei et al. ${ }^{38}$ demonstrated the use of diffraction patterns of a submerged superhydrophobic plasma desorption mass spectrometry (PDMS) grating to characterize the wetting state and transitions on micropillars, although only qualitative information was obtained. More quantitative measurements of the wetting depth and wetting transitions on microstructures have been reported by Saad ${ }^{39}$ and Dufour ${ }^{40}$ by analysis of the reflection of high-frequency acoustic waves at the solid-liquid interface. Wetting characterization of nanostructures is still under exploration. Conventional optical methods fail on the nanoscale because of resolution limitations, and imaging of the liquid-solid interface on the nanoscale typically requires environmental ${ }^{29}$ or cryobased $^{30}$ scanning electron microscopy (SEM). Several techniques have been recently developed, based on indirect measurements of a physical property that can be correlated with the wetting state. Using ordered arrays of silicon nanopillars, we have demonstrated that nanoscale liquid penetration depths can be accurately determined using optical reflectance spectroscopy. ${ }^{13,41}$ Wetting transitions and gas trapping on the same nanopillars with superhydrophobic coatings have also been quantitatively characterized using an acoustic method ${ }^{26}$ and an attenuated total reflectance-Fourier transform infrared (ATRFTIR)-based technique to detect trapping of gaseous carbon dioxide in between the pillars. ${ }^{27}$ Most of the above mentioned techniques can be used to probe a small local area, but for material characterizations, a method suitable for in situ, largearea wetting characterization is still needed.

ATR-FTIR is a well-known surface characterization technique to identify substances and reactions near a surface. Its high surface sensitivity and nondestructive character makes it especially popular for the analysis of biological samples. ${ }^{42,43}$ ATR-FTIR relies on total internal reflection of an infrared (IR) beam inside of an ATR-crystal. An evanescent wave is emitted every time the beam is reflected and penetrates outside the crystal with an electric field amplitude that decays in an exponential manner. ${ }^{44-46}$ The molecules in the sample absorb the evanescent waves with wavelengths corresponding to the excitation energies of their vibration modes, resulting in characteristic absorption bands in the spectrum. The sensitivity and the measurement area can be increased by multiple reflections of the IR beam inside of the crystal. ${ }^{45,47}$ A detailed mathematical study on the nature and physical interpretation of the evanescent wave has recently been discussed in the work of Milosevic. ${ }^{46}$ To assess the decay of the evanescent wave, Harrick and du Pré ${ }^{48}$ defined the "penetration depth" as the sample depth for which the electric field amplitude of the evanescent wave, $E$, decays to a value of $E_{0} \mathrm{e}^{-1}$ ( $E_{0}$ is the value of the electric field amplitude on the surface). Penetration depths of IR radiation are generally on the order of a few hundreds of nanometers, rendering ATR-FTIR very wellsuited to explore the liquid-solid and air-solid interactions on the nanoscale. For example, Öhman ${ }^{49,50}$ and Taheri ${ }^{51,52}$ applied ATR-FTIR for the in situ analysis of water penetration through organic coatings on top of a metal layer. Exploitation of the highly surface-sensitive character of this method enabled them to monitor various surface and interface phenomena including oxide formation, evolution of the $\mathrm{OH}$-termination, degradation of the interfacial bonding, and water diffusion toward the surface.

In this work, we demonstrate a novel ATR-FTIR-based method for the in situ analysis of the wetting state of silicon nanopillars. First, contact angle measurements are used to measure the surface wettability using isopropanol (IPA) and water mixtures. Then, a detailed nanoscale wetting characterization is carried out using ATR-FTIR. The wetting stability and the wetting hysteresis are discussed at the end.

\section{EXPERIMENTS}

Surface Treatments. Dense arrays of silicon nanopillars (height $260 \pm 9 \mathrm{~nm}$, diameter $40 \pm 3 \mathrm{~nm}$, and pitch $90 \mathrm{~nm}$ ) are fabricated using deep ultraviolet (UV) immersion lithography and plasma etching technology, as described in previous works. ${ }^{12,13,16,26,27,53}$ The dimensions and the mechanical stability of the structures are assessed using SEM. The pillars are sufficiently robust to withstand the capillary force-induced aggregation or collapse upon wetting and drying, as illustrated in Figure S5. The hydrophobicity of the surface is tuned through dry surface-modification techniques, that is, $\mathrm{UV} / \mathrm{O}_{3}$-treatment and coating with $1 \mathrm{H}, 1 \mathrm{H}, 2 \mathrm{H}, 2 \mathrm{H}$-perfluorodecyl-trichlorosilane (FDTS). Experimental details are given in the Supporting Information. $\mathrm{UV} / \mathrm{O}_{3}$-treatment renders the surface hydrophilic (contact angle $<5^{\circ}$ on flat $\mathrm{Si}(100)$ and silicon pillars) by removing the organic contamination from the surface. FDTS deposition yields superhydrophobic surfaces, with a static contact angle of $110^{\circ}$ on flat $\mathrm{Si}(100)$ and an apparent contact angle of $148^{\circ}$ on the patterned samples. The superhydrophobicity of similar FDTS-coated nanostructures has also been confirmed in our previous works, using highfrequency acoustics and optical reflectance methods. ${ }^{13,26}$

Goniometric Measurements. $\mathrm{UV} / \mathrm{O}_{3}$-treated samples have contact angles below $5^{\circ}$ and show complete wetting in between the structures for water and IPA. Contact angle measurements are performed on both FDTS-coated silicon nanopillars and FDTS-coated flat silicon samples, using premixed mixtures of water and IPA. The apparent contact angles measured on the patterned substrates as a function of the static contact angles on the flat substrates are shown in Figure 1 for various IPA concentrations. The contact angles monotonically decrease upon addition of IPA, owing to the reduction in the liquid surface tension $\left(\gamma_{\mathrm{IPA}}=21 \mathrm{mN} / \mathrm{m}\right.$ and $\left.\gamma_{\mathrm{H}_{2} \mathrm{O}}=72 \mathrm{mN} / \mathrm{m}\right)$. Pure IPA exhibits a contact angle of $53 \pm 3^{\circ}$ on flat surfaces and $30 \pm$ $5^{\circ}$ on patterned substrates, clearly corresponding to the Wenzel state. Two zones can be observed in Figure 1, corresponding to Wenzel wetting at a high IPA concentration and Cassie-Baxter wetting at a low IPA concentration, separated by a clear discontinuity in the slope. Therefore, decreasing the surface tension induces a transition from the 


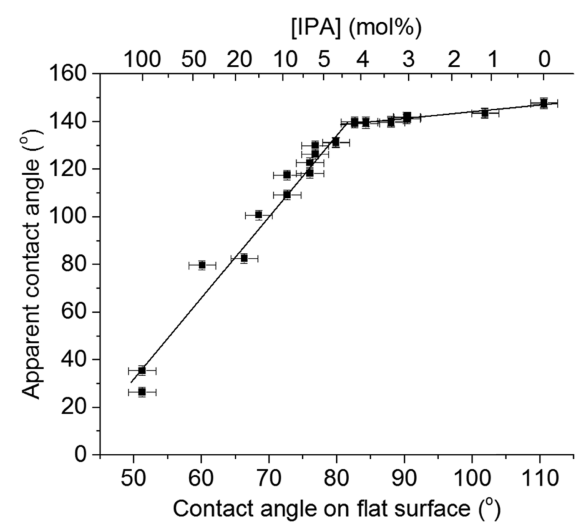

Figure 1. Contact angle measurements of IPA-water mixtures on FDTS-coated silicon nanopillars. The pillars are $260 \pm 9 \mathrm{~nm}$ high and have a diameter of $40 \pm 3 \mathrm{~nm}$. The black lines are added to guide the eye.

Cassie-Baxter state to the Wenzel state once a certain critical value has been reached. The transition point is situated around $4.0 \mathrm{~mol} \%$ of IPA. The breakdown of the Cassie-Baxter wetting states occurs still in the hydrophilic regime with contact angles on flat surfaces around $82^{\circ}$. This value is significantly lower than that predicted by the transition criterion obtained from the classical wetting models described by Wenzel and Cassie and Baxter ${ }^{13}\left(\sim 99^{\circ}\right.$, obtained from eqs 1 and 2 with $r=5.4$ and $\left.f_{\mathrm{s}}=0.16\right)$. The observations are in very good agreement with our previous works, ${ }^{13,27}$ in which the deviation was attributed to fabrication-induced surface alterations such as mixed crystal planes and atomic-scale lattice defects originating from random ion bombardments or atomic-scale inhomogeneous chemical etching.

A steep transition from the Cassie-Baxter to Wenzel state is resolved from the goniometric measurements based on the analysis of macroscopic droplets. However, the nanoscale interactions at the liquid-air interface and the liquid behavior near the center of the droplet remain open questions. In the light of this, ATR-FTIR is used to gain additional insights into the nanoscale wetting phenomena, owing to its excellent surface sensitivity and feasibility of large-area sampling.

ATR-FTIR. An FTIR spectrometer is equipped with a customized flow cell, as schematically illustrated in Figure 2. ATR-crystals (crystal

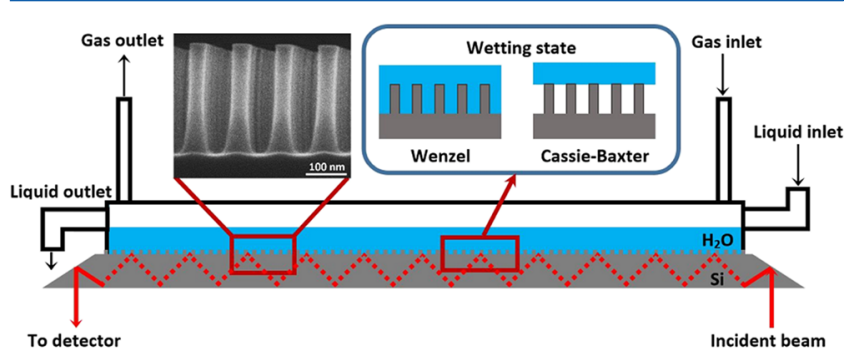

Figure 2. Schematic representation of the ATR-FTIR setup.

dimensions: $5.5 \times 3.0 \mathrm{~cm}$, thickness $775 \mu \mathrm{m}$, crystal angle $30^{\circ}$, and angle of incidence $60^{\circ}$ ) are prepared from blanket silicon wafers and from silicon wafers patterned with nanopillars by mechanical polishing. Two conditions should be satisfied to achieve total reflection inside of the crystal: ${ }^{44,45}(1)$ the refractive index of the crystal $\left(n_{1}\right)$ should be larger than the refractive index of the sample $\left(n_{2}\right)$ and $(2)$ the angle of incidence $(\theta)$ should exceed the critical angle $\theta_{\mathcal{c}}$ defined as $\theta_{c}=\sin ^{-1}$ $\left(n_{2} / n_{1}\right)$. These conditions are met in the experiments $\left(\theta_{\mathrm{c}} \approx 22^{\circ}, n_{\mathrm{Si}}=\right.$ $3.45, n_{\mathrm{H}_{2} \mathrm{O}}=1.33$, and $\left.n_{\text {air }}=1.00\right)$. The combination of a relatively low solid-surface fraction (0.16) and pillar height on the order of the evanescent wave penetration depth ensures good experimental sensitivity to any liquid or gas in between the structures. The IR beam is internally total reflected 18 times in the crystal to enhance the signal intensity. In the experiments, before the liquid is injected in the cell, a reference spectrum is collected in air. This reference spectrum is later subtracted from the spectra recorded in the liquids. Thus, any bands originating from different surface functionalization are removed, and the obtained IR absorption spectra reflect only the changes induced by the wetting of the ATR-crystal. The resolution is $2.0 \mathrm{~cm}^{-1}$ with a short acquisition time of only $5 \mathrm{~s}$ to reduce the impact of evaporation-induced change on the mixture composition. Spectrum acquisition was started within $5 \mathrm{~s}$ after liquid injection. A more elaborate description of the experimental procedure is given in the Supporting Information.

Wetting States of Pure Water. Water absorption spectra are compared for flat and nanopillared silicon crystals with $\mathrm{UV} / \mathrm{O}_{3^{-}}$or FDTS-treatment (Figure 3). Two absorption bands are present at

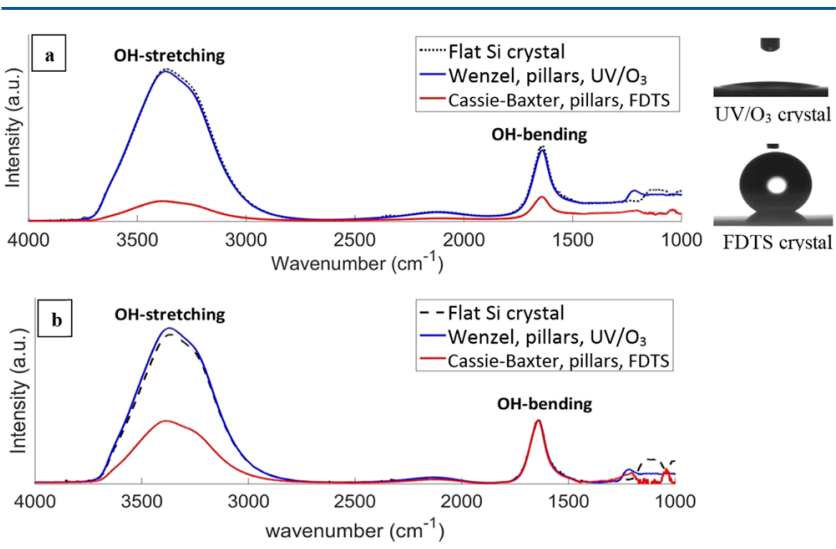

Figure 3. FTIR spectra of pure water recorded on a hydrophilic UV/ $\mathrm{O}_{3}$-treated flat silicon reference crystal (dotted black), a hydrophilic $\mathrm{UV} / \mathrm{O}_{3}$-treated crystal patterned with silicon nanostructures (blue), and a superhydrophobic FDTS-coated crystal patterned with silicon nanostructures (red). The liquid on the hydrophilic patterned crystal is in the Wenzel state, whereas it is in the Cassie-Baxter state on the superhydrophobic crystal. (a) Spectra before baseline correction and normalization, clearly showing the decrease in the spectrum intensity for the Cassie-Baxter state compared with that of the Wenzel state. Images of water drops on both crystals are given in the right inset. (b) Spectra after baseline correction and normalization with respect to the $\mathrm{OH}$-bending peak. The ratio between the water peak intensities equals $2.6 \pm 0.1$ for the Wenzel state and $1.0 \pm 0.1$ for the Cassie-Baxter state.

1640 and $3380 \mathrm{~cm}^{-1}$ corresponding to the $\mathrm{OH}$-bending and $\mathrm{OH}-$ stretching modes of water, respectively. ${ }^{54}$ Water spectra recorded on $\mathrm{UV} / \mathrm{O}_{3}$-treated silicon nanopillars are similar to the spectra recorded on a flat surface. By contrast, the spectra recorded on the superhydrophobic nanopillars coated with FDTS significantly differ from the fully wetted samples by an overall decrease in the spectrum intensity by 5-10 times (Figure 3a). As water is in the Cassie-Baxter state on these nanopillars, an air layer is present in between the structures. This air layer causes additional attenuation of the evanescent wave, which accounts for the observed decrease in the signal intensity.

The water spectra recorded on the flat silicon crystals and hydrophilic $\mathrm{UV} / \mathrm{O}_{3}$-treated nanopillars are characterized by a relative peak intensity ratio $I_{\mathrm{OH}-\text { stretching }} / I_{\mathrm{OH}-\text { bending }}$ of $2.6 \pm 0.1$. This ratio is obtained after baseline correction and normalization with respect to the OH-bending peak, as illustrated in Figure 3b. It is not significantly affected upon changing the surface chemistry of the flat crystal, as illustrated in the Supporting Information for three different surface chemistries (Figure S1). The normalized spectrum of water in the Cassie-Baxter state clearly deviates from the Wenzel spectrum, and the relative peak intensity ratio $I_{\mathrm{OH}-\text { stretching }} / I_{\mathrm{OH} \text {-bending }}$ decreases to $\approx 1.0$ \pm 0.1 . The penetration depth of the evanescent wave $\left(d_{\mathrm{p}}\right)$ is inversely proportional to the wavenumber $\nu$ according to eq $3^{44,45,47}$ 


$$
d_{\mathrm{p}}=\frac{1}{2 \pi \nu \sqrt{n_{1}^{2} \sin ^{2} \theta-n_{2}^{2}}}
$$

where $\nu$ represents the wavenumber of the incident radiation, $n_{1}$ and $n_{2}$ are the refractive indices of the ATR-crystal and the sample, respectively, and $\theta$ is the angle of the incident wave. At 1640 and $3380 \mathrm{~cm}^{-1}$, the IR light penetration depths in water are equal to 0.363 and $0.176 \mu \mathrm{m}$, respectively. In the case of evanescent waves propagating in air, these values decrease by approximately $5 \%$ compared with propagation in water. It should be remarked that after traveling a distance $d_{\mathrm{p}}$ into the sample, the electric field amplitude of the evanescent wave decayed to a value $E_{0} \mathrm{e}^{-1}$, and thus, the penetration depth defined in eq 3 does not correspond to the entire sampled depth. The latter one can be approximated by $3 d_{\mathrm{p}}$, but most of the signal is obtained from shallower depths. ${ }^{44}$ The spectrum intensities will be attenuated more at high wavenumbers by an additional air layer compared with that at lower wavenumbers. This results in a reduced relative intensity ratio between the $\mathrm{OH}$-stretching and $\mathrm{OH}$-bending bands, from $\sim 2.6 \pm 0.1$ for the Wenzel wetting state to $\sim 1.0 \pm 0.1$ for the Cassie-Baxter wetting state. The proposed method is solely based on the wavelength dependency in evanescent wave attenuation, and it allows for unambiguous differentiation between the Wenzel and Cassie-Baxter states on patterned surfaces. It should be remarked that various precautions are taken to limit the generation of air bubbles on the crystal: (1) all experiments are performed in a cleanroom environment using ultrapure water, (2) sample preparation is carefully handled with fresh surface treatment and cleaning before the experiment to avoid organic contaminants, and (3) special care is taken during liquid injection to avoid bubble entrainment. Macroscopic bubbles or uncovered crystal areas were never observed during the experiments, but micro-/nanobubbles may exist after water injection. In the experiments, the obtained intensity ratios of the 2 water bands are very reproducible, with a standard deviation of approximately $4 \%$ for more than 55 measurements. It is therefore postulated that nanobubbles do not play any critical role because otherwise, more nonreproducible fluctuations in the measurements are expected.

Wetting State of IPA-Water Mixtures. Premixed mixtures of water and IPA with increasing IPA concentrations are injected in the liquid cell. The recorded spectra are shown in Figure 4. IPA also shows an $\mathrm{OH}$-stretching band around $3380 \mathrm{~cm}^{-1}$, although less intense compared with that of water. The sharp absorption band around 2930 $\mathrm{cm}^{-1}$ corresponds to the stretching mode of the $\mathrm{CH}$-bonds. Other characteristic peaks are found at lower wavenumbers (below 1500 $\mathrm{cm}^{-1}$ ) but are not used for quantitative analysis because of the strong silicon absorption and the resultant high noise level in this range. The spectra of deionized water (DIW)/IPA mixtures show three main bands, that is, the $\mathrm{OH}$-stretching peak from both IPA and DIW, the $\mathrm{CH}$-stretching band originating from IPA, and the OH-bending band characteristic of DIW. The relative intensities of these bands depend on the mixture composition and can thus be used for a quantitative analysis of the IPA content in the solution. This will be elaborated in the Discussion section.

The spectra recorded on the $\mathrm{UV} / \mathrm{O}_{3}$-treated nanopillars are shown in Figure 4a. All mixtures are in the Wenzel state on these nanopillars, as can be seen from the side-view images of sessile drops in the inset. A monotonically decreasing trend is observed for the intensities of the $\mathrm{OH}$-stretching and $\mathrm{OH}$-bending bands because of the decrease in the water content of the mixture, and there is an increase in the $\mathrm{CH}$ stretching band intensities owing to the increase in the IPA concentration.

The spectra recorded on the superhydrophobic FDTS-coated nanopillars are depicted in Figure 4b. IPA concentrations below 2.8 mol \% result in a 5-10 fold decrease in the spectrum intensity compared with that of the $\mathrm{UV} / \mathrm{O}_{3}$-treated reference. The spectra in this concentration range are similar to the spectrum of pure water in the Cassie-Baxter state (Figure 3), with a small $\mathrm{CH}$-stretching peak superimposed, resulting from the presence of IPA. Therefore, IPA mixtures with concentrations below $2.8 \mathrm{~mol} \%$ are in the CassieBaxter state on the FDTS-coated nanopillars. The spectra for IPA
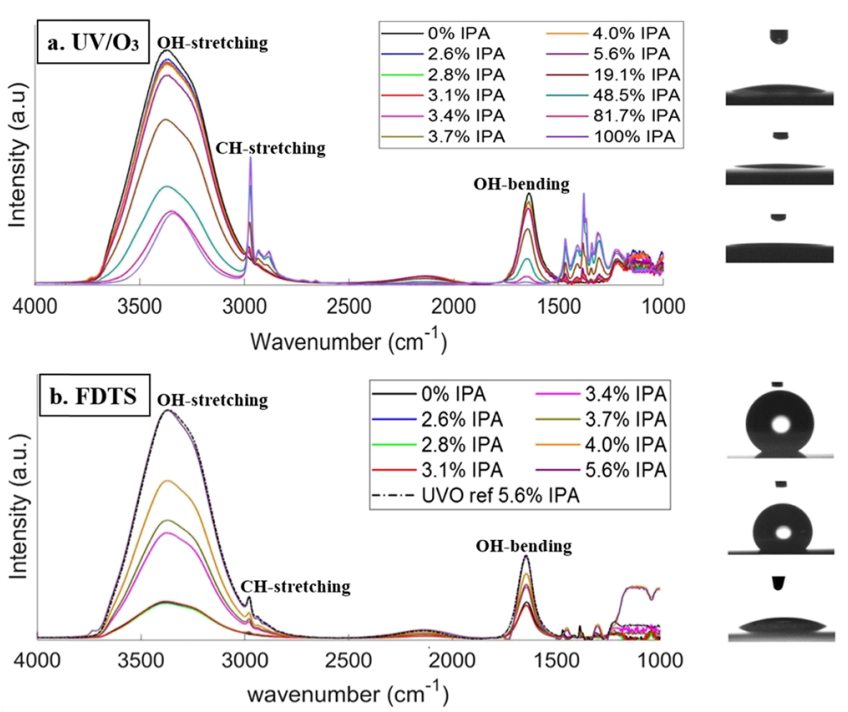

Figure 4. (a) Spectra of IPA-water mixtures recorded on hydrophilic $\mathrm{UV} / \mathrm{O}_{3}$-treated nanopillars. IPA concentrations depicted in the graph range from 0 to $100 \mathrm{~mol} \%$. The right inset depicts the contact angles on the $\mathrm{UV} / \mathrm{O}_{3}$-treated crystal for pure water (top), pure IPA (bottom), and a mixture of $5.6 \mathrm{~mol} \%$ IPA (middle). (b) Spectra of IPA-water mixtures recorded on superhydrophobic FDTS-coated nanopillars. The right inset depicts the contact angles for pure water (top), pure IPA (bottom), and a mixture of $5.6 \mathrm{~mol} \%$ IPA (middle). The spectra for concentrations above $5.6 \mathrm{~mol} \%$ do not differ from the ones recorded for the $\mathrm{UV} / \mathrm{O}_{3}$-treated crystals thus are not added to the graph for clarity.

concentrations above $4.0 \mathrm{~mol} \%$ are identical to the ones recorded on the $\mathrm{UV} / \mathrm{O}_{3}$-treated crystal, indicating a Wenzel state. The results are in excellent agreement with the data from the contact angle measurements, where also a transition around $4.0 \mathrm{~mol} \%$ was found. The spectra recorded in the concentration ranges below $2.8 \mathrm{~mol} \%$ or above $4.0 \mathrm{~mol} \%$ are reproducible and stable in time (measurement ranges up to $2 \mathrm{~h}$ ).

At intermediate concentrations (2.8-4.0 mol \%), the recorded spectra on the FDTS-coated nanopillars show a substantial increase in the intensities of the $\mathrm{OH}$-bending and $\mathrm{OH}$-stretching peaks compared with those at lower IPA concentrations. This evolution is not consistent with the earlier observations of a decrease in the water bands for mixtures with increasing IPA concentration. The recorded spectra also clearly differ from their counterparts recorded on the hydrophilic crystals, which have much higher spectrum intensity. Therefore at this intermediate concentration range, these spectra correspond neither to pure Wenzel nor to pure Cassie-Baxter states but are attributed to mixed wetting states. The experimental reproducibility is poor in this range, with large variations in measurements because of local fluctuations. The spectra are found to evolve in time (timescale several tens of minutes up to few hours), with a tendency to converge to the hydrophilic reference spectra. The observations suggest a dynamic transition from the Cassie-Baxter state to the Wenzel state. Further study of the kinetics is out of the scope of this work.

\section{DISCUSSION}

Wetting States. The presence of an air layer in between the structures was shown to induce two significant changes in the FTIR spectrum of pure water: (1) decrease in the overall spectrum intensity by a factor of 5-10 and (2) a reduction in the relative intensity ratio of the $\mathrm{OH}$-stretching peak and the OH-bending peak from $2.6 \pm 0.1$ (Wenzel) to $1.0 \pm 0.1$ (Cassie-Baxter). This peak intensity ratio, $I_{\mathrm{OH}-\text { stretching }} /$ $I_{\mathrm{OH} \text {-bending, }}$ can yield a more accurate estimation of the wetting 
state and is less sensitive to experimental variations that affect the absolute spectrum intensity. However, in the presence of IPA, the ratio can be affected by the IPA concentration in the mixture because both water and IPA contribute to the $\mathrm{OH}$ stretching peak. The variation in the peak intensity ratio as a function of the IPA concentration on $\mathrm{UV} / \mathrm{O}_{3}$-treated nanopillars is depicted in Figure 5. A significant increase is found

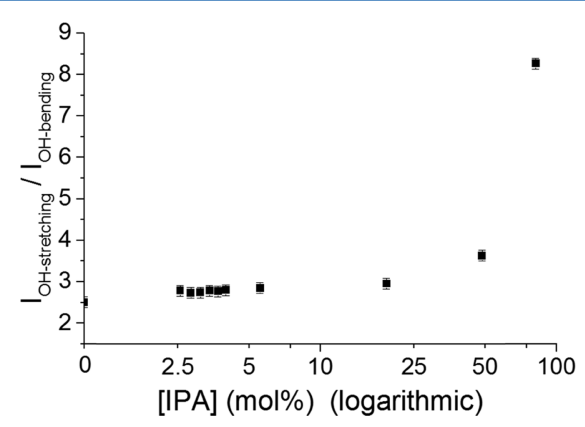

Figure 5. Evolution of the relative intensity ratio of the water bands as a function of the IPA concentration. For Wenzel states of IPA-water mixtures on $\mathrm{UV} / \mathrm{O}_{3}$-treated nanopillars, the relative peak intensity ratio of the $\mathrm{OH}$-stretching and $\mathrm{OH}$-bending peaks is almost constant around 2.6 for IPA concentrations below $20 \mathrm{~mol} \%$.

only for IPA concentrations above $20 \mathrm{~mol} \%$, owing to the diminishing OH-bending peak for low water contents. For IPA concentrations below $20 \mathrm{~mol} \%$, the peak intensity ratio is found to be roughly constant around $2.6 \pm 0.1$ and hence can be used as a reference value for the assessment of the wetting state.

The evolution of the relative intensity ratio of the water peaks as a function of the IPA concentration is depicted in Figure 6 for both superhydrophobic FDTS-coated and

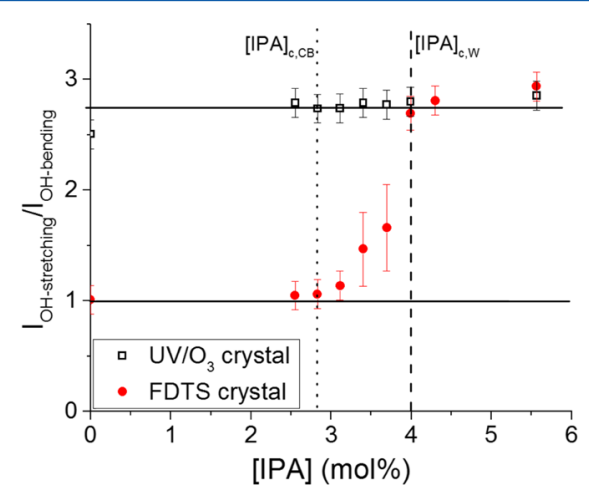

Figure 6. Variation in the relative intensity ratio of the $\mathrm{OH}$-stretching and $\mathrm{OH}$-bending peaks as a function of the IPA concentration on hydrophilic (UV/O $/ \mathrm{O}_{3}$-treated, black squares) and superhydrophobic (FDTS-deposited, red circles) patterned crystals. The dashed lines correspond to the maximum IPA concentration for which the liquid is still in a stable Cassie-Baxter state $\left([\mathrm{IPA}]_{\mathrm{c}, \mathrm{CB}}\right)$ and the minimum IPA concentration for which the Wenzel state is stable $\left([\mathrm{IPA}]_{c, W}\right)$.

hydrophilic $\mathrm{UV} / \mathrm{O}_{3}$-treated nanopillars. There is a clear difference between the Wenzel and Cassie-Baxter states as well as mixed wetting states. The FDTS-coated nanopillars exhibit a superhydrophobic behavior for pure DIW and IPAwater mixtures below $2.8 \mathrm{~mol} \%$, denoted as $[\mathrm{IPA}]_{\mathrm{c}, \mathrm{CB}}$ (surface tension $38.8 \mathrm{mN} / \mathrm{m}$ ), and a Wenzel behavior for mixtures with IPA concentrations above $4.0 \mathrm{~mol} \%$, denoted as $[\mathrm{IPA}]_{c, \mathrm{~W}}$ (surface tension $34.3 \mathrm{mN} / \mathrm{m}$ ). The surface tension values for the different IPA mixtures are tabulated in Table S1. The results are consistent with the work of $\mathrm{Li}$ et al., in which highfrequency acoustic methods were used to determine the wetting state of DIW-ethanol mixtures. ${ }^{26}$ They reported a critical ethanol concentration of $27.5 \mathrm{vol} \%$ (8.2 mol \%) for superhydrophobic breakdown, corresponding to a critical surface tension of $38.2 \mathrm{mN} / \mathrm{m}$ on similar nanostructures. Mixtures with concentrations ranging from $[\mathrm{IPA}]_{\mathrm{c}, \mathrm{W}}$ to $[\mathrm{IPA}]_{\mathrm{c}, \mathrm{CB}}$ exhibit peak intensity ratios between 1 and 2.6, which do not correspond to any of the pure wetting states. They represent mixed wetting states and are observed to evolve in time. This suggests a transient process of slow liquid penetration in between the structures and breaking-down of the superhydrophobic state. The relatively large measurement errors on the initial values of the relative peak intensity ratio for these data points are attributed to the extremely unstable character of the Cassie-Baxter state in this concentration range. Local transitions can be easily triggered by a variety of largely uncontrollable phenomena: (1) local fluctuations in IPA concentrations, (2) variation in the FDTS packing density, (3) local defects in the structure arrays, and (4) local pressure variation during liquid injection. The developed method allows for qualitative comparison regarding the amount of liquid penetration.

It should be noted that only one sharp transition around 4.0 mol \% IPA was found with contact angle measurements. However, with ATR-FTIR, mixed wetting states in the range of $2.8-4.0 \mathrm{~mol} \%$ IPA can also be distinguished. Therefore, ATR-FTIR can be a more accurate technique for nanomaterial characterization because of the high surface sensitivity and large-area measurement.

Wetting Hysteresis. The feasibility of inducing a transition from the Cassie-Baxter state to the Wenzel state by increasing the IPA concentration has been demonstrated. Another intriguing aspect is to find out whether the transition can be reversed by lowering the IPA concentration again. The study of wetting hysteresis and reversibility of the wetting state yields valuable insights into the energy landscape. In situ analysis of such processes requires adequate characterization methods to capture the dynamic changes in the liquid composition and wetting state simultaneously. Most techniques proposed in the literature cannot accommodate these combined effects. Owing to its high surface sensitivity, ATR-FTIR can be a perfect technique to characterize the wetting hysteresis.

Analysis of the IPA content is based on the relative intensities of the $\mathrm{CH}$-stretching and $\mathrm{OH}$-stretching bands. Mixtures with known compositions are used to establish a calibration curve. A linear correlation with a slope of $0.019 \pm 0.0003$ per mol \% is found between the IPA concentration and the relative intensity ratio of the $\mathrm{CH}$ - and $\mathrm{OH}$-stretching bands (Figure 7). This approach yields a good estimation of the IPA concentration with an error of $\pm 0.4 \mathrm{~mol} \%$ in the range of interest. The fitting is not significantly affected by the surface chemistry and surface patterning, as illustrated in Figure S2. Changes in the wetting state may slightly alter the calibration curve because of a relative difference in the attenuation between the $\mathrm{CH}$-stretching and $\mathrm{OH}$-stretching bands. However, as these bands are relatively close to each other in the spectrum, such side effects are found negligible. This composition measurement is thus applicable to both flat and patterned substrates and does not require significant corrections when altering the surface chemistry or surface roughness. 


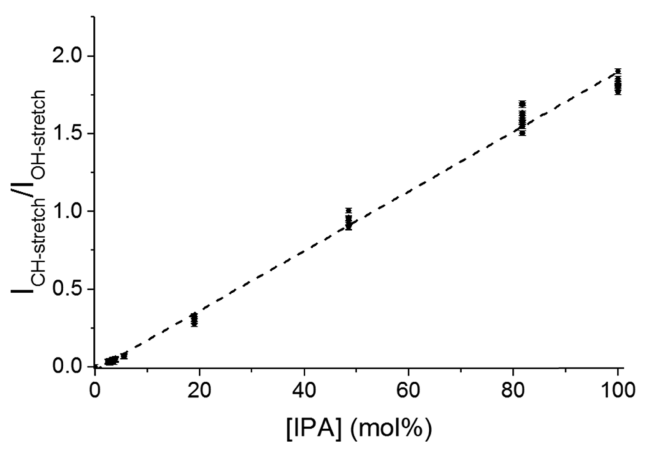

Figure 7. Calibration curve for the determination of the IPA concentration near the surface. Measurements are performed with a resolution of $2.0 \mathrm{~cm}^{-1}$ and an acquisition time of $14 \mathrm{~s}$.

After the superhydrophobic state breaks down at higher IPA concentrations, experiments are conducted to study the stability of the Wenzel state as the IPA concentration is gradually decreased by (1) evaporation or (2) dilution with pure water. The results from both experimental sets are summarized in Figure 8. Evaporation experiments are conducted starting from

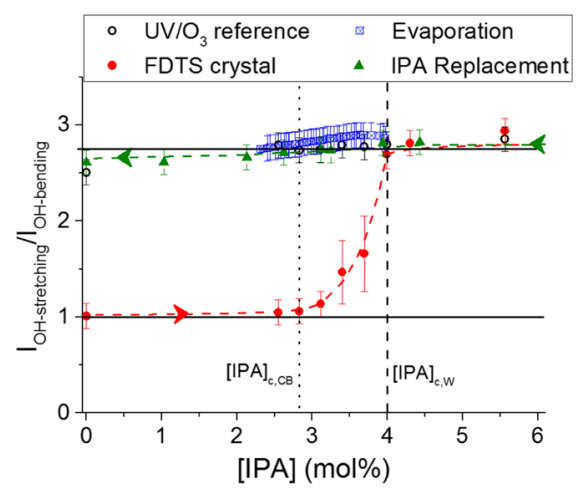

Figure 8. Wetting hysteresis. The data points marked with solid red circles show the transition from the Cassie-Baxter to Wenzel states upon increasing the IPA concentration on superhydrophobic FDTScoated crystals. After a Wenzel state has been established, the IPA concentration is gradually lowered by evaporation (open blue squares) and water rinsing (solid green triangles). The dotted lines are added to guide the eye. They indicate the observed trends upon increasing (red line) and decreasing (green line) the IPA concentration.

a $4.0 \mathrm{~mol} \%$ mixture over a time-lapse of $2 \mathrm{~h}$ with a $\mathrm{N}_{2}$ gas flow of $180 \mathrm{ccm}$. IPA replacement experiments were started from a crystal wetted with pure IPA, and then IPA was replaced by flushing with pure water $(1.25 \mathrm{~mL} / \mathrm{s})$. No IPA peak could be detected anymore after approximately $90 \mathrm{~s}$. The raw data corresponding to the individual experiments are given in Figures S3 and S4. The dashed red curve in Figure 8 shows the breakdown of superhydrophobicity upon increasing the IPA concentration, as was discussed previously (Figure 6). The dashed green line represents the observed trend when the IPA concentration is decreased again either by evaporation (squares) or dilution (triangles). The relative peak intensity ratio initially corresponds to the characteristic ratio for the Wenzel state $(2.6 \pm 0.1)$ and roughly remains constant. The minor decrease for very low IPA concentrations is attributed to the higher water content in the mixture, consistent with the data summarized in Figure 5. Consequently, water can be brought in the Wenzel state on superhydrophobic surfaces. The obtained state is stable in time, and any further alterations of the surface tension cannot induce spontaneous dewetting toward the Cassie-Baxter state.

The observation of a large wetting hysteresis is in agreement with other experimental works on the reversibility of the Cassie-Baxter-to-Wenzel transition..$^{10,11,31,55-58}$ Transitions from the Cassie-Baxter to Wenzel state are widely illustrated in the literature using various approaches including gravitational effects, ${ }^{21,55,59}$ mechanical pressure, ${ }^{24,38,60}$ addition of lower surface tension solvents, ${ }^{26,56,61}$ evaporation of droplets, ${ }^{40,62}$ bouncing of droplets, ${ }^{63,64}$ vibration, ${ }^{63,65}$ and modification of the surface chemistry. ${ }^{13}$ By contrast, wetting transitions from the Wenzel to Cassie-Baxter state on nanopillars cannot occur spontaneously but require a large external energy input, for example, through local laser heating till the Leidenfrost temperature $^{57}$ or application of an external voltage. ${ }^{66}$ This suggests a higher energy barrier for the transition from the Wenzel to Cassie-Baxter state. Many recent works $3,10,11,67-72$ using free-energy calculations found similar energy paths, with the Wenzel state having a lower Gibb's free energy than the Cassie-Baxter state on patterned surfaces. This implies Cassie-Baxter states to be metastable on most nonhierarchical structures. Additionally, as these transitions are dynamic processes, contact angle hysteresis may also play an important role in the reversibility of the transition. Both aspects, that is, the height of the energy barrier and the contact angle hysteresis will contribute to an increased resistance to dewetting of the structures.

\section{CONCLUSIONS}

In this work, we have developed a novel ATR-FTIR-based method, capable of in situ monitoring of wetting states on large-area nanostructured surfaces over long time-ranges (few seconds to few hours). Differentiation between the Wenzel and Cassie-Baxter wetting states and an analysis of partial wetting is based on the water spectrum and the variation in the relative peak intensity ratio between the $\mathrm{OH}$-stretching and $\mathrm{OH}$ bending peaks. This ratio is highly sensitive to the wetting state, owing to the wavelength dependency in the evanescent wave attenuation and is not affected by the surface functionalization. The developed ATR-FTIR method is expected to be applicable to a wide range of pillar heights. In the case of pure Wenzel states, the water spectrum is not affected by the patterning of the sample. Thus, the relative intensity ratio of the water bands will remain to be $2.6 \pm 0.1$ irrespective of the pillar height and profile. For pure Cassie-Baxter states, there should be a minimum pillar height, below which the attenuation of evanescent waves by the trapped air layer is too small to be distinguished from the absorption spectra. The exact numbers of this minimum height depend on the sensitivity of the FTIR system used in experiments and will not be commented here. On the other hand, increasing the pillar height will enhance the air-induced attenuation, and the relative peak intensity ratio of the water bands is expected to decrease even further. For pillar heights above the sampling depths (500-1000 nm), the evanescent wave will completely be attenuated by the air layer in between the pillars, and no more water signal can be acquired. In that case, it is still feasible to distinguish between pure Wenzel and pure Cassie-Baxter states because the water spectrum for the Wenzel state remains unchanged.

This ATR-FTIR technique is used to study the wetting transitions and the wetting hysteresis. On superhydrophobic nanopillars, a transition from the Cassie-Baxter to Wenzel 
states is induced by increasing the IPA concentrations of IPAwater mixtures. The breakdown of superhydrophobicity and the existence of mixed wetting states were observed for solutions with surface tensions in the range of $34-39 \mathrm{mN} / \mathrm{m}$. Mixtures with surface tensions above $39 \mathrm{mN} / \mathrm{m}$ were found to be in the Cassie-Baxter state, whereas solutions with surface tensions below $34 \mathrm{mN} / \mathrm{m}$ resulted in complete wetting of the structures. ATR-FTIR could resolve mixed wetting states occurring across the crystal, which were not captured by goniometric measurements. A large wetting hysteresis was found, and water could be achieved in a stable Wenzel state on the originally superhydrophobic surfaces. This ATR-FTIR technique can be used to monitor both the wetting state and the liquid composition near the surface in real time and can be very promising for studying the wetting kinetics on nanostructures, which will be elaborated in a future work.

\section{ASSOCIATED CONTENT}

\section{S Supporting Information}

The Supporting Information is available free of charge on the ACS Publications website at DOI: 10.1021/acs.langmuir.6b04471.

Materials and methods; molar fraction and surface tensions for different mixtures of IPA and ethanol; evaluation of the effects of surface chemistry and surface patterning on the relative intensity ratio of the water bands, variation in the relative intensity ratio of the water peaks for different surface chemistries $\left[\mathrm{UV} / \mathrm{O}_{3}\right.$-treated, coated with FDTS and coated with phenethyltrichlorosilane (PETS)] on flat crystals and for a crystal patterned with silicon nanopillars; evaluation of the effects of surface chemistry and surface patterning on the measured IPA concentration, as calculated from the calibration curve; wetting hysteresis, evaporation of a 4.0 mol \% IPA mixture, replacement of pure IPA by pure water on a FDTS-coated crystal patterned with silicon nanopillars; and assessment of the mechanical integrity of the pillars after a wetting experiment (PDF)

\section{AUTHOR INFORMATION}

\section{Corresponding Authors}

*E-mail: Nandi.vrancken@imec.be (N.V.).

*E-mail: XiuMei@imec.be (X.X.).

\section{ORCID}

Nandi Vrancken: 0000-0003-2461-0252

\section{Present Address}

"Imec, Kapeldreef 75, 3001 Leuven, Belgium (S.S.).

\section{Author Contributions}

The manuscript was written through contributions of all authors. All authors have given approval to the final version of the manuscript.

\section{Funding}

N.V. acknowledges the Fonds voor Wetenschappelijk Onderzoek (FWO) for a research grant.

Notes

The authors declare no competing financial interest.

\section{ABBREVIATION}

IPA, isopropanol

\section{REFERENCES}

(1) Wenzel, R. N. Resistance of solid surfaces to wetting by water. Ind. Eng. Chem. 1936, 28, 988-994.

(2) Cassie, A. B. D.; Baxter, S. Wettability of porous surfaces. Trans. Faraday Soc. 1944, 40, 546-551.

(3) Quéré, D. Wetting and Roughness. Annu. Rev. Mater. Res. 2008, $38,71-99$.

(4) Quéré, D. Rough ideas on wetting. Phys. A 2002, 313, 32-46.

(5) Bico, J.; Tordeux, C.; Quéré, D. Rough wetting. Europhys. Lett. 2001, 55, 214-220.

(6) Barthlott, W.; Neinhuis, C. Purity of the sacred lotus, or escape from contamination in biological surfaces. Planta 1997, 202, 1-8.

(7) Ragesh, P.; Ganesh, V. A.; Nair, S. V.; Nair, A. S. A review on "self-cleaning and multifunctional materials". J. Mater. Chem. A 2014, 2, 14773-14797.

(8) Krishna, M. G.; Vinjanampati, M.; Purkayastha, D. D. Metal oxide thin films and nanostructures for self-cleaning applications: Current status and future prospects. Eur. Phys. J.: Appl. Phys. 2013, 62, 30001.

(9) Wu, W.; Cheng, L.; Yuan, M.; Bai, S.; Wei, Z.; Jing, T.; et al. Surface Engineering Method to Fabricate a Bendable Self-Cleaning Surface with High Robustness. Sci. Adv. Mater. 2013, 5, 933-938.

(10) Bormashenko, E. Progress in understanding wetting transitions on rough surfaces. Adv. Colloid Interface Sci. 2015, 222, 92-103.

(11) Bormashenko, E. Wetting transitions on biomimetic surfaces. Philos. Trans. R. Soc., A 2010, 368, 4695-4711.

(12) Xu, X. M.; Vereecke, G.; van den Hoogen, E.; Smeers, J.; Armini, S.; Delande, T.; et al. Wetting Challenges in Cleaning of High Aspect Ratio Nano-Structures. Solid State Phenom. 2012, 195, 235238.

(13) Xu, X.; Vereecke, G.; Chen, C.; Pourtois, G.; Armini, S.; Verellen, N.; et al. Capturing Wetting States in Nanopatterned Silicon. ACS Nano 2014, 8, 885-893.

(14) Hiramoto, T.; Miyaji, K.; Kobayashi, M. Nanoscale Silicon Devices Using Nanostructure Physics for VLSI Applications. In Fifth Hiroshima International Workshop on Nanoelectronics for Tera-Bit Information Processing, Campus Innovation Center, Tokyo (Japan), 29-30 January 2007, pp 29-30.

(15) Kuhn, K. J. CMOS Transistor Scaling Past $32 \mathrm{~nm}$ and Implications on Variation. In Advanced Semiconductor Manufacturing Conference and Workshop. Proceedings of the IEEE/Semi Advanced Semiconductor Manufacturing Conference; IEEE, 2010; pp 241-246.

(16) Li, J.; Chen, C.; Jans, H.; Xu, X.; Verellen, N.; Vos, I.; et al. 300 $\mathrm{mm}$ Wafer-level, ultra-dense arrays of Au-capped nanopillars with sub$10 \mathrm{~nm}$ gaps as reliable SERS substrates. Nanoscale 2014, 6, 1239112396.

(17) Chen, C.; Xu, X.; Li, Y.; Jans, H.; Neutens, P.; Kerman, S.; et al. Full wetting of plasmonic nanopores through two-component droplets. Chem. Sci. 2015, 6, 6564-6571.

(18) Kim, B. S.; Shin, S.; Shin, S. J.; Kim, K. M.; Cho, H. H. Control of Superhydrophilicity/Superhydrophobicity using Silicon Nanowires via Electroless Etching Method and Fluorine Carbon Coatings. Langmuir 2011, 27, 10148-10156.

(19) Dorrer, C.; Rühe, J. Wetting of Silicon Nanograss: From Superhydrophilic to Superhydrophobic Surfaces. Adv. Mater. 2008, 20, $159-163$.

(20) Jung, Y. C.; Bhushan, B. Dynamic Effects of Bouncing Water Droplets on Superhydrophobic Surfaces. Langmuir 2008, 24, 62626269.

(21) Martines, E.; Seunarine, K.; Morgan, H.; Gadegaard, N.; Wilkinson, C. D. W.; Riehle, M. O. Superhydrophobicity and Superhydrophilicity of Regular Nanopatterns. Nano Lett. 2005, 5, 2097-2103.

(22) Krupenkin, T. N.; Taylor, J. A.; Schneider, T. M.; Yang, S. From Rolling Ball to Complete Wetting: The Dynamic Tuning of Liquids on Nanostructured Surfaces. Langmuir 2004, 20, 3824-3827.

(23) Kuo, C. Y.; Gau, C. Control of Superhydrophilicity and Superhydrophobicity of a Superwetting Silicon Nanowire Surface. J. Electrochem. Soc. 2010, 157, K201-K205. 
(24) Lafuma, A.; Quéré, D. Superhydrophobic states. Nat. Mater. 2003, 2, 457-460.

(25) Gao, L.; Mccarthy, T. J. Wetting $101^{\circ}$. Langmuir 2009, 25, $14105-14115$.

(26) Li, S.; Lamant, S.; Carlier, J.; Toubal, M.; Campistron, P.; Xu, X.; et al. High-Frequency Acoustic for Nanostructure Wetting Characterization. Langmuir 2014, 30, 7601-7608.

(27) Vereecke, G.; Xu, X.; Tsai, W.-K.; Yang, H.; Armini, S.; Delande, T.; et al. Wetting Behavior of Aqueous Solutions on High Aspect Ratio Nanopillars with Hydrophilic Surface Finish. ECS Trans. 2013, 58, 171-182.

(28) Papadopoulos, P.; Mammen, L.; Deng, X.; Vollmer, D.; Butt, H.-J. How superhydrophobicity breaks down. Proc. Natl. Acad. Sci. U.S.A. 2013, 110, 3254-3258.

(29) Paxson, A. T.; Varanasi, K. K. Self-similarity of contact line depinning from textured surfaces. Nat. Commun. 2013, 4, 1492-1498. (30) Rykaczewski, K.; Landin, T.; Walker, M. L.; Scott, J. H. J.; Varanasi, K. K. Direct Imaging of Complex Nano- to Microscale Interfaces Involving Solid, Liquid, and Gas Phases. ACS Nano 2012, 6, 9326-9334.

(31) Verho, T.; Korhonen, J. T.; Sainiemi, L.; Jokinen, V.; Bower, C.; Franze, K.; et al. Reversible switching between superhydrophobic states on a hierarchically structured surface. Proc. Natl. Acad. Sci. U.S.A. 2012, 109, 10210-10213.

(32) Sbragaglia, M.; Peters, A. M.; Pirat, C.; Borkent, B. M.; Lammertink, R. G. H.; Wessling, M.; et al. Spontaneous Breakdown of Superhydrophobicity. Phys. Rev. Lett. 2007, 99, 156001.

(33) Dorrer, C.; Rühe, J. Condensation and Wetting Transitions on Microstructured Ultrahydrophobic Surfaces. Langmuir 2007, 23, $3820-3824$

(34) Schellenberger, F.; Xie, J.; Encinas, N.; Hardy, A.; Klapper, M.; Papadopoulos, P.; et al. Direct observation of drops on slippery lubricant-infused surfaces. Soft Matter 2015, 11, 7617-7626.

(35) Schellenberger, F.; Encinas, N.; Vollmer, D.; Butt, H.-J. How Water Advances on Superhydrophobic Surfaces. Phys. Rev. Lett. 2016, 116, 096101.

(36) Lv, P.; Xue, Y.; Shi, Y.; Lin, H.; Duan, H. Metastable States and Wetting Transition of Submerged Superhydrophobic Structures. Phys. Rev. Lett. 2014, 112, 196101.

(37) Moulinet, S.; Bartolo, D. Life and death of a fakir droplet: Impalement transitions on superhydrophobic surfaces. Eur. Phys. J. E: Soft Matter Biol. Phys. 2007, 24, 251-260.

(38) Lei, L.; Li, H.; Shi, J.; Chen, Y. Diffraction Patterns of a WaterSubmerged Superhydrophobic Grating under Pressure. Langmuir 2010, 26, 3666-3669.

(39) Saad, N.; Dufour, R.; Campistron, P.; Nassar, G.; Carlier, J.; Harnois, M.; et al. Characterization of the state of a droplet on a micro-textured silicon wafer using ultrasound. J. Appl. Phys. 2012, 112, 104908.

(40) Dufour, R.; Saad, N.; Carlier, J.; Campistron, P.; Nassar, G.; Toubal, M.; et al. Acoustic Tracking of Cassie to Wenzel Wetting Transitions. Langmuir 2013, 29, 13129-13134.

(41) Gwon, M.; Kim, S.; Li, J.; Xu, X.; Kim, S.-K.; Lee, E.; et al. Influence of wetting state on optical reflectance spectra of Si nanopillar arrays. J. Appl. Phys. 2015, 118, 213102.

(42) Glassford, S. E.; Byrne, B.; Kazarian, S. G. Recent applications of ATR FTIR spectroscopy and imaging to proteins. Biochim. Biophys. Acta, Proteins Proteomics 2013, 1834, 2849-2858.

(43) Kazarian, S. G.; Chan, K. L. A. Applications of ATR-FTIR spectroscopic imaging to biomedical samples. Biochim. Biophys. Acta 2006, 1758, 858-867.

(44) Mirabella, F. Internal Reflection Spectroscopy: Theory and Applications; CRC Press: New York, 1992.

(45) Harrick, N. J. Internal Reflection Spectroscopy, 3rd ed.; Harrick Scientific Corporation; New York, 1967.

(46) Milosevic, M. On the Nature of the Evanescent Wave. Appl. Spectrosc. 2013, 67, 126-131.

(47) Harrick, N. J. Surface chemistry from spectral analysis of totally internally reflected radiation. J. Phys. Chem. 1960, 64, 1110-1114.
(48) Harrick, N. J.; du Pré, F. K. Effective Thickness of Bulk Materials and of Thin Films for Internal Reflection Spectroscopy. Appl. Opt. 1966, 5, 1739-1743.

(49) Öhman, M.; Persson, D.; Leygraf, C. A Spectroelectrochemical Study of Metal/Polymer Interfaces by Simultaneous in Situ ATR-FTIR and EIS. Electrochem. Solid-State Lett. 2007, 10, C27-C30.

(50) Öhman, M.; Persson, D. An integrated in situ ATR-FTIR and EIS set-up to study buried metal-polymer interfaces exposed to an electrolyte solution. Electrochim. Acta 2007, 52, 5159-5171.

(51) Taheri, P.; Flores, J. R.; Hannour, F.; de Wit, J. H. W.; Terryn, H.; Mol, J. M. C. In Situ Study of Buried Interfacial Bonding Mechanisms of Carboxylic Polymers on Zn Surfaces. J. Phys. Chem. C 2013, 117, 3374-3382.

(52) Taheri, P.; de Wit, J. H. W.; Terryn, H.; Mol, J. M. C. In Situ Study of Buried Metal-Polymer Interfaces Exposed to an Aqueous Solution by an Integrated ATR-FTIR and Electrochemical Impedance Spectroscopy System. J. Phys. Chem. C 2013, 117, 20826-20832.

(53) Vos, I.; Hellin, D.; Vertommen, J.; Demand, M.; Boullart, W. Silicon Nano-Pillar Test Structures for Quantitative Evaluation of Wafer Drying Induced Pattern Collapse. ECS Trans. 2011, 41, 189196.

(54) Coker, D. F.; Reimers, J. R.; Watts, R. O. The Infrared Absorption Spectrum of Water. Aust. J. Phys. 1982, 35, 623-638.

(55) Peters, A. M.; Pirat, C.; Sbragaglia, M.; Borkent, B. M.; Wessling, M.; Lohse, D.; Lammertink, R. G. H. Cassie-Baxter to Wenzel state wetting transition: Scaling of the front velocity. Eur. Phys. J. E: Soft Matter Biol. Phys. 2009, 29, 391-397.

(56) Murakami, D.; Jinnai, H.; Takahara, A. Wetting Transition from the Cassie-Baxter State to the Wenzel State on Textured Polymer Surfaces. Langmuir 2014, 30, 2061-2067.

(57) Liu, G.; Fu, L.; Rode, A. V.; Craig, V. S. J. Water Droplet Motion Control on Superhydrophobic Surfaces: Exploiting the Wenzel-to-Cassie Transition. Langmuir 2011, 27, 2595-2600.

(58) Bahadur, V.; Garimella, S. V. Electrowetting-Based Control of Droplet Transition and Morphology on Artificially Microstructured Surfaces. Langmuir 2008, 24, 8338-8345.

(59) Jung, Y. C.; Bhushan, B. Wetting transition of water droplets on superhydrophobic patterned surfaces. Scr. Mater. 2007, 57, 10571060.

(60) Forsberg, P.; Nikolajeff, F.; Karlsson, M. Cassie-Wenzel and Wenzel-Cassie transitions on immersed superhydrophobic surfaces under hydrostatic pressure. Soft Matter 2011, 7, 104-109.

(61) Koch, B. M. L.; Amirfazli, A.; Elliott, J. A. W. Wetting of Rough Surfaces by a Low Surface Tension Liquid. J. Phys. Chem. C 2014, 118, 23777-23782.

(62) Tsai, P.; Lammertink, R. G. H.; Wessling, M.; Lohse, D. Evaporation-Triggered Wetting Transition for Water Droplets upon Hydrophobic Microstructures. Phys. Rev. Lett. 2010, 104, 116102.

(63) Jung, Y. C.; Bhushan, B. Dynamic Effects Induced Transition of Droplets on Biomimetic Superhydrophobic Surfaces. Langmuir 2009, 25, 9208-9218.

(64) Bartolo, D.; Bouamrirene, F.; Verneuil, É.; Buguin, A.; Silberzan, P.; Moulinet, S. Bouncing or sticky droplets: Impalement transitions on superhydrophobic micropatterned surfaces. Europhys. Lett. 2006, 74, 299-305.

(65) Bormashenko, E.; Pogreb, R.; Whyman, G.; Erlich, M. CassieWenzel Wetting Transition in Vibrating Drops Deposited on Rough Surfaces: Is the Dynamic Cassie-Wenzel Wetting Transition a 2D or 1D Affair? Langmuir 2007, 23, 6501-6503.

(66) Vrancken, R. J.; Kusumaatmaja, H.; Hermans, K.; Prenen, A. M.; Pierre-Louis, O.; Bastiaansen, C. W. M.; et al. Fully Reversible Transition from Wenzel to Cassie-Baxter States on Corrugated Superhydrophobic Surfaces. Langmuir 2010, 26, 3335-3341.

(67) Giacomello, A.; Meloni, S.; Chinappi, M.; Casciola, C. M. Cassie-Baxter and Wenzel States on a Nanostructured Surface: Phase Diagram, Metastabilities, and Transition Mechanism by Atomistic Free Energy Calculations. Langmuir 2012, 28, 10764-10772.

(68) Giacomello, A.; Chinappi, M.; Meloni, S.; Casciola, C. M. Superhydrophobicity lost: The Cassie-Baxter to Wenzel phase 
transition. In Proceedings of the XXI Congresso AIMETA, Torina (Italy),

17-20 September 2013.

(69) Patankar, N. A. On the Modeling of Hydrophobic Contact Angles on Rough Surfaces. Langmuir 2003, 19, 1249-1253.

(70) Patankar, N. A. Transition between Superhydrophobic States on Rough Surfaces. Langmuir 2004, 20, 7097-7102.

(71) Patankar, N. A. Consolidation of Hydrophobic Transition Criteria by Using an Approximate Energy Minimization Approach. Langmuir 2010, 26, 8941-8945.

(72) Marmur, A. Wetting on Hydrophobic Rough Surfaces: To Be Heterogeneous or Not To Be? Langmuir 2003, 19, 8343-8348. 